\title{
Aspectos bionômicos de Eois tegularia (Guenée) e Eois glauculata (Walker) (Lepidoptera, Geometridae, Larentiinae) e seus parasitóides ${ }^{1}$
}

\author{
Sandra Maria Pasenow Braga ${ }^{2}$ \\ Manoel Martins Dias ${ }^{3}$ \\ Angélica Maria Penteado-Dias ${ }^{3}$
}

\begin{abstract}
Bionomic aspects of Eois tegularia (Guenée) and Eois glauculata (Walker) (Lepidoptera, Geometridae, Larentiinae) and their parasitoids. Eois tegularia (Guenée, [1858]) and Eois glauculata (Walker, [1863]) larvae were studied by one year in semideciduous forest at Canchim Farm (EMBRAPA), São Carlos, São Paulo, Brazil. Specimens were hosted by species of Piper Linnaeus (Piperaceae): $P$. mollicomum Kunth, $P$. amalago Linnaeus, $P$. glabratum Kunth and $P$. gaudichaudianum Kunth. Some parasitoids were reared from the larvae of Eois Hübner, 1818 species, as follow. Hymenoptera: Cotesia Cameron, 1891, Apanteles Foerster, 1862, Glyptapanteles Ashmead, 1904, Dolichogenidea Viereck, 1911, Meteorus Haliday, 1835 (Braconidae); Mesochorus Gravenhorst, 1829, Venturia Schrottky, 1902 (Ichneumonidae); Elachertus Spinola, 1811 (Eulophidae); Perilampus Latreille, 1809 (Perilampidae). Diptera: Myiopharus Brauer \& Bergenstamm, 1889 (Tachinidae).

KEY WORDS. Lepidoptera, Geometridae, Eois, Piperaceae, Hymenoptera, Diptera, parasitoids
\end{abstract}

Foram estudadas Eois tegularia (Guenée, [1858]) e Eois glauculata (Walker, [1863]) (Geometridae, Larentiinae), cujas larvas desenvolveram-se sobre Piperaceae, em mata semidecídua com 117 hectares (800-900 m de altitude) da Fazenda Canchim (EMBRAPA), São Carlos, São Paulo, Brasil. A denominação das espécies de Geometridae seguiu SCOBLE (1999); este autor mencionou Croton sylvaticus Hochst. (Euphorbiaceae) como planta hospedeira de Eois grataria (Walker, 1861).

MARQUIS (1990) referiu-se a Eois Hübner, 1818 causando danos foliares em Piper arieianum C.D. ex Pittier (Piperaceae), na Costa Rica, onde essa planta abriga numerosa fauna de herbívoros. MARQUIS (1991) mencionou em La Selva, Costa Rica, 21 espécies de Larentiinae sobre folhas de Piper Linnaeus; muitos dos Geometridae por ele observados foram especialistas quanto à planta hospedeira.

1) Realizado com auxilio do Programa BIOTA/FAPESP e CAPES.

2) Programa de Pós-Graduação em Ecologia e Recursos Naturais, Universidade Federal de São Carlos. Caixa Postal 676, 13565-905 São Carlos, São Paulo, Brasil.

3) Departamento de Ecologia e Biologia Evolutiva, Universidade Federal de São Carlos. Caixa Postal 676, 13565-905 São Carlos, São Paulo, Brasil. 


\section{MATERIAL E MÉTODOS}

Larvas foram obtidas no interior da mata, por meio de guarda-chuva entomológico, em caminho de $500 \mathrm{~m}$ de comprimento e 2-3 m de largura. As Piperaceae ocorrem com relativa abundância nas bordas desse caminho, representadas por plantas de 1-2 m de altura. Os trabalhos foram mensais, no período de julho/1995 a junho/1996. Larvas coletadas foram mantidas em laboratório sobre as respectivas plantas hospedeiras, até a obtenção dos imagos ou parasitóides.

\section{RESULTADOS}

Quatro espécies de Piperaceae hospedaram larvas de E. tegularia e E. glauculata: Piper mollicomum Kunth, Piper amalago Linnaeus, Piper glabratum Kunth e Piper gaudichaudianum Kunth.

Das larvas obtidas de E. tegularia, 44,5\% estavam sobre P. mollicomum; de E. glauculata, 52,6\% estavam sobre $P$. amalago. O período com maior ocorrência de larvas nas Piperaceae, foi de abril a julho, após as chuvas, quando houve maior brotação das espécies de Piper e portanto maior disponibilidade de alimento e abrigo para as larvas de Eois. Nesse período foram registrados $71,8 \%$ das larvas de E. tegularia e $80,3 \%$ de E. glauculata (Tabs I-II); esse número declinou em julho e depois, acentuadamente, até outubro, período correspondente à seca na região.

Foram constatados os seguintes parasitóides, obtidos de larvas de $E$. tegularia e E. glauculata. Hymenoptera: Cotesia Cameron, 1891, Apanteles Foerster, 1862, Glyptapanteles Ashmead, 1904, Dolichogenidea Viereck, 1911, Meteorus Haliday, 1835 (Braconidae); Mesochorus Gravenhorst, 1829, Venturia Schrottky, 1902 (Ichneumonidae); Elachertus Spinola, 1811 (Eulophidae); Perilampus Latreille, 1809 (Perilampidae). Diptera: Myiopharus Brauer \& Bergenstamm, 1889 (Tachinidae).

Datas de ocorrência e número de exemplares dos parasitóides estão nas tabelas III e IV. Glyptapanteles sp. ocorreu em larvas de E. tegularia coletadas sobre todas as espécies de Piper, e também em larvas de E. glauculata, sobre P. amalago. Também Myiopharus sp. ocorreu com frequiência nas larvas estudadas; foi o parasitóide com maior número de exemplares registrados. Não foi constatado parasitismo sobre larvas de E. glauculata obtidas em $P$. glabratum e $P$. gaudichaudianum. Aproximadamente $50 \%$ dos parasitóides de E. tegularia foram representados por Microgastrinae. Mesochorus sp. e Perilampus sp. são hiperparasitóides, sendo obtidos indiretamente através de larvas de Eois spp. TOWNES \& TOWNES (1966) citaram Mesochorus havrylenkoi Havrylenko \& Winterhalter, 1949 como parasitóide em larva de Geometridae, na Argentina; segundo DASCH (1974) essa espécie denomina-se Mesochorus discitergus (Say, 1836).

AgRAdecimentos. Ao Centro de Pesquisa de Pecuária do Sudeste (EMBRAPA), pelo acesso e facilidades relativas à área de estudo. Ao Dr. Waldir Mantovani pela identificação das espécies de Piperaceae; ao Dr. José Henrique Guimarães pela identificação do diptero parasitóide. 
Tabela I. Eois tegularia. Número de larvas nas diferentes espécies de Piper e respectivos meses de ocorrência em 1995 e 1996.

\begin{tabular}{|c|c|c|c|c|c|c|c|c|c|c|c|c|c|}
\hline \multirow{2}{*}{ Species } & \multicolumn{6}{|c|}{1995} & \multicolumn{6}{|c|}{1996} & \multirow{2}{*}{ Total } \\
\hline & Jul & Ago & Set & Out & Nov & Dez & Jan & $\mathrm{Fev}$ & Mar & Abr & Mai & Jun & \\
\hline Piper mollicomum & 54 & 24 & 1 & 1 & 4 & 3 & 8 & 23 & 17 & 24 & 49 & 48 & 256 \\
\hline Piper amalago & 29 & 4 & 1 & 2 & 1 & 0 & 1 & 3 & 3 & 5 & 36 & 28 & 113 \\
\hline Pieper glabratum & 6 & 33 & 0 & 0 & 0 & 0 & 1 & 0 & 2 & 11 & 3 & 11 & 67 \\
\hline Piper gaudichaudianum & 13 & 0 & 0 & 0 & 0 & 1 & 8 & 13 & 8 & 16 & 27 & 53 & 139 \\
\hline
\end{tabular}

Tabela II. Eois glauculata. Número de larvas nas diferentes espécies de Piper e respectivos meses de ocorrência em 1995 e 1996.

\begin{tabular}{|c|c|c|c|c|c|c|c|c|c|c|c|c|c|}
\hline \multirow{2}{*}{ Species } & \multicolumn{6}{|c|}{1995} & \multicolumn{6}{|c|}{1996} & \multirow{2}{*}{ Total } \\
\hline & Jul & Ago & Set & Out & Nov & Dez & Jan & Fev & Mar & $\mathrm{Abr}$ & Mai & Jun & \\
\hline Piper mollicomum & 18 & 6 & 1 & 0 & 0 & 0 & 1 & 1 & 13 & 6 & 18 & 18 & 82 \\
\hline Piper amalago & 11 & 1 & 2 & 1 & 2 & 0 & 3 & 3 & 9 & 27 & 60 & 33 & 152 \\
\hline Pieper glabratum & 1 & 4 & 0 & 0 & 0 & 0 & 0 & 0 & 4 & 3 & 1 & 2 & 15 \\
\hline Piper gaudichaudianum & 2 & 1 & 0 & 0 & 0 & 1 & 0 & 0 & 4 & 2 & 17 & 13 & 40 \\
\hline
\end{tabular}

Tabela III. Parasitóides obtidos de larvas de Eois tegularia nas diferentes espécies de Piper e respectivos meses de ocorrência em 1995 e 1996.

\begin{tabular}{|c|c|c|c|}
\hline & Parasitóides & $\begin{array}{l}\text { Data de coleta e número de } \\
\text { exemplares (entre parênteses) }\end{array}$ & $\begin{array}{l}\text { Número total de } \\
\text { parasitóides }\end{array}$ \\
\hline \multirow[t]{4}{*}{ Piper mollicomum } & Microgastrinae* & $04 / 96(1), 06 / 96(1)$ & 2 \\
\hline & Apanteles sp. & $08 / 95(1)$ & 1 \\
\hline & Glyptapanteles sp. & $11 / 95(1), 05 / 96(4)$ & 5 \\
\hline & Myiopharus sp. & $07 / 95(1), 12 / 95(1), 04 / 96(1), 06 / 96(1)$ & 4 \\
\hline \multirow[t]{7}{*}{ Piper amalago } & Microgastrinae* & $06 / 96(2)$ & 2 \\
\hline & Glyptapanteles sp. & $08 / 95(1), 05 / 96(1)$ & 2 \\
\hline & Dolichogenidea sp. & $05 / 96(2), 06 / 96(2)$ & 4 \\
\hline & Meteorus sp. & $06 / 96(1)$ & 1 \\
\hline & Mesochorus sp. & $08 / 95(1)$ & 1 \\
\hline & Venturia sp. & $08 / 95(1)$ & 1 \\
\hline & Elachertus sp. & $05 / 96(4)$ & 4 \\
\hline \multirow[t]{6}{*}{ Piper glabratum } & Microgastrinae* & $07 / 95(2), 06 / 96(2)$ & 4 \\
\hline & Glyptapanteles sp. & $08 / 95(3)$ & 3 \\
\hline & Meteorus sp. & $04 / 96(2), 05 / 96(1)$ & 3 \\
\hline & Venturia sp. & $04 / 96(2)$ & 2 \\
\hline & Elachertus sp. & $04 / 96(3)$ & 3 \\
\hline & Myiopharus sp. & $08 / 95(11), 06 / 96(3)$ & 14 \\
\hline \multirow[t]{4}{*}{ Piper gaudichaudianum } & Microgastrinae* & $01 / 96(1), 04 / 96(1), 05 / 96(1)$ & 3 \\
\hline & Cotesia sp. & $01 / 96(1)$ & 1 \\
\hline & Glyptapanteles sp. & $06 / 96(1)$ & 1 \\
\hline & Myiopharus sp. & $03 / 96(1), 06 / 96(1)$ & 2 \\
\hline
\end{tabular}

$\left.{ }^{*}\right)$ Microgastrinae (Braconidae): individuos machos identificados apenas ao nivel de subfamilia. 
Tabela IV. Parasitóides obtidos de larvas de Eois glauculata nas diferentes espécies de Piper e respectivos meses de ocorrência, em 1995 e 1996.

\begin{tabular}{lllc}
\hline Parasitóides & \multicolumn{1}{c}{$\begin{array}{c}\text { Data de coleta e número de } \\
\text { exemplares (entre parènteses) }\end{array}$} & $\begin{array}{c}\text { Número total de } \\
\text { parasitóides }\end{array}$ \\
\hline Piper mollicomum & $\begin{array}{l}\text { Microgastrinae * } \\
\text { Venturia sp. }\end{array}$ & $\begin{array}{l}07 / 95(2), 03 / 96(1), 06 / 96(1) \\
07 / 95(1)\end{array}$ & 4 \\
& & & 1 \\
Piper amalago & Microgastrinae * & $05 / 96(1)$ & 1 \\
& Dolichogenidea sp. & $05 / 96(2)$ & 2 \\
& Glyptapanteles sp. & $06 / 96(1)$ & 1 \\
& Meteorus sp. & $06 / 96(1)$ & 1 \\
& Elachertus sp. & $05 / 96(1), 06 / 96(9)$ & 1 \\
\hline
\end{tabular}

${ }^{*}{ }^{*}$ Microgastrinae (Braconidae): individuos machos identificados apenas ao nivel de subfamilia.

\section{REFERÊNCIAS BIBLIOGRÁFICAS}

DASCH, C.E. 1974. Neotropic Mesochorinae (Hymenoptera: Ichneumonidae). Mem. Amer. Ent. Inst. 22: $1-509$.

MARQUUS, R.J. 1990. Genotypic variation in leaf damage in Piper arieianum (Piperaceae) by a multispecies assemblage of herbivores.

Evolution 44 (1): 104-120.

1991. Herbivore fauna of Piper (Piperaceae) in a Costa Rican wet forest: diversity, specificity and impact, p. 179-208. In: P.W. Price; T.M. LeWINSOHN; G.W. FerNANDES \& W.W. BENSON (Eds). Plant-animal interations: evolutionary ecology in tropical and temperate regions. New York, J. Wiley \& Sons Inc., XIV+639p.

SCOBLE, M.J. 1999. Geometrid moths of the world. A catalogue. Collingwood, CSIRO Publ., Vol. 1, $\mathrm{XXV}+482 \mathrm{p}$.

TOWNES, H. \& M. TOWNES. 1966. A catalogue and reclassification of the Neotropic Ichneumonidae. Mem. Amer. Ent. Inst. 8: I-III+ 1-367.

Recebido em 03.VIII.2000; aceito em 02.VIII.2001. 